Int. J. Contemp. Math. Sciences, Vol. 2, 2007, no. 5, 233 - 239

\title{
On the Degree of Theta Pairs of Finite Groups ${ }^{1}$
}

\author{
A. Erfanian and R. Rezaei \\ Department of Mathematics \\ Faculty of Mathematical Sciences \\ Ferdowsi University of Mashhad, Mashhad, Iran \\ erfanian@math.um.ac.ir, ras_rezaei@yahoo.com
}

\begin{abstract}
Let $G$ be a finite group and $M$ a maximal subgroup of $G$. A $\theta$-pair of $M$ is any pair of subgroups $(C, D)$ of $G$ such that (i) $D \triangleleft G, D<C$, (ii) $\langle M, C\rangle=G,\langle M, D\rangle=M$ and (iii) $\frac{C}{D}$ has no proper normal subgroup of $\frac{G}{D}$. A $\theta^{\star}$-pair of $M$ is a pair of subgroups $(C, D)$ satisfying conditions (i) and (iii) and a property that $D \leq M$ and $C^{g} \nsubseteq M$ for every $g \in G$.

In this paper, we introduce the degree of $\theta$-pairs, denoted by $d \theta(G)$ as the ratio $|\theta(G)| / m(G)$, where $\theta(G)$ is the union of all $\theta$-pairs of the maximal subgroups of $G$ and $m(G)$ is the total number of distinct maximal subgroups of $G$. Similarly, we define the degrees of maximal $\theta$-pairs, $\theta^{\star}$-pairs and maximal $\theta^{\star}$-pairs of a finite group $G$ and give some evaluations on the above degrees for some simple groups, nilpotent groups and solvable groups. Moreover, we prove that if $G$ is nilpotent then the degree of maximal $\theta$-pairs of $G$ is exactly 1 .
\end{abstract}

Keywords: $\theta$-pair, $\theta^{\star}$-pair, maximal $\theta$-pair, maximal $\theta^{\star}$-pair, degree of $\theta$-pair, degree of $\theta^{\star}$-pair

Mathematics Subject Classification: 20D10,20E34.

\section{Introduction}

In 1990, Mukhrejee and Bhattacharya [4] introduced the notion of $\theta$-pair for every maximal subgroup of a finite group. They have proved the existance of

\footnotetext{
${ }^{1}$ The authors thank the Centre of Excellency in Analysis on Algebraic Structures, Ferdowsi University of Mashhad for supporting the part grant on this work.
} 
a $\theta$-pair for any maximal subgroup $M$ of a finite group $G$. Two years later, Beidleman and Smith [2], generalized the concept of $\theta$-pair for infinite groups. In [8], Yaoqing improved the definition of $\theta$-pair for a maximal subgroup of a finite group $G$ to a maximal $\theta$-pair and normal $\theta$-pair. He proved that for any maximal subgroup $M$ of a finite group $G$, there exists a normal maximal $\theta$-pair related to $M$. The investigationon $\theta$-pair are continued by others in $[1,7-9]$. Most of ths research deals with the structure of finite groups. Later, in 2000, Shirong and Yaoqing in [6], defined the new concept $\theta^{\star}$-pair which is a special case of $\theta$-pairs by some additional conditions. Using the concept of $\theta^{\star}$-pair, they obtained several results on maximal $\theta^{\star}$-pairs which imply a finite group $G$ to be solvable or supersolvable.

In this paper, we introduced a degree on the $\theta$-pairs of a finite group $G$ which is called $\theta$-pairs degree of $G$ and will be stated later. In this article, we prove that if $G$ is a finite nilpotent group, then the $\theta$-pairs and $\theta^{\star}$-pairs degrees are exactly 1 . Moreover, we will compare the above degrees of a group $G$ with the quotient group $\frac{G}{N}$, where $N$ is a normal subgroup of $G$ and contained in the Frattini subgroup $\Phi(G)$. Now we give some definitions and basic results.

\section{Some definitions and basic results}

All groups considered in this paper, are assumed to be finite. We also denote $M \underset{\max }{<} G$ as a convenience that $M$ is a maximal subgroup of $G$. Now, we start with the definition of $\theta$-pair given by Mukhrejee and Bhattocharya in [4].

Definition 2.1 Let $G$ be a finite group and $M$ a maximal subgroup $G$. A $\theta$-pair of $M$ is any pair $(C, D)$ of subgroups satisfying the following conditions

(a) $D \triangleleft G, D<C$.

(b) $\langle M, C\rangle=G$ and $\langle M, D\rangle=M$.

(c) $\frac{C}{D}$ has no proper normal subgroup of $\frac{G}{D}$.

Definition 2.2 Let $(C, D)$ be a $\theta$-pair of a maximal subgroup $M$ of $G$. Then $(C, D)$ is called a normal $\theta$-pair if $C \triangleleft G$ and is said to be a maximal $\theta$-pair if there is no $\theta$-pair $\left(C^{\prime}, D^{\prime}\right)$ such that $C<C^{\prime}$.

The set of all $\theta$-pairs of a maximal subgroup $M$ of $G$ is denoted by $\theta(M)$. We also denote $\theta(G)$ as the union of $\theta(M)$ for all maximal subgroups $M$ of $G$. Similarly, $\theta_{m}(M)$ and $\theta_{m}(G)$ will be denoted for those of $\theta$-pairs which are maximal.

It is clear that for every maximal subgroup $M$ of $G, \theta(M)$ and $\theta_{m}(M)$ are not empty. Since $\left(C, M_{G}\right)$ is a $\theta$-pair of $M$, where $M_{G}$ is the core of $M$ in $G$ and $C$ is a subgroup of $G$ such that $\frac{C}{M_{G}}$ is a chief factor of $G$. It is easy to see that $\left(C, M_{G}\right)$ is a maximal $\theta$-pair of $M$ as well. 
Definition 2.3 given a maximal subgroup $M$ of a finite group $G$, a $\theta^{\star}$-pair of $M$ is any pair $(C, D)$ of subgroup of $G$ such that

(a) $D<C$ and $D \triangleleft G$,

(b) $D \leq M$ but $C^{g} \nsubseteq M$ for every $g \in G$ and

(c) $\frac{C}{D}$ has no proper normal subgroup of $\frac{G}{D}$.

Similar to those of $\theta$-pairs, we can define the concepts : normal, maximal $\theta^{\star}$-pair and the notations $\theta^{\star}(M), \theta_{m}^{\star}(M)$ and $\theta^{\star}(G)$.

By the above two definitions, we can see that every $\theta^{\star}$-pair of $M$ is a $\theta$-pair of $M$ and so $\theta^{\star}(M) \subseteq \theta(M)$. Thus, we have $\theta^{\star}(G) \subseteq \theta(G)$. It is also clear that a normal $\theta^{\star}$-pair is certainly maximal and $\theta^{\star}$-pair $\left(M^{g}\right)=\theta^{\star}$-pair $(\mathrm{M})$ for every maximal subgroup $M$ of $G$ and every $g \in G$.

In the rest of this section, we state some known results on the $\theta$-pairs and $\theta^{\star}$-pairs. We omitt the proofs and one may find them in [4].

Theorem 2.4 If $(C, D)$ is a maximal $\theta$-pair in $\theta(M)$ and $N \triangleleft G, N<D$, then $\left(\frac{C}{N}, \frac{D}{N}\right)$ is a maximal $\theta$-pair in $\theta\left(\frac{M}{N}\right)$. Conversly, if $\left(\frac{C}{N}, \frac{D}{N}\right)$ is a maximal $\theta$-pair in $\theta\left(\frac{M}{N}\right)$, then $(C, D)$ is a maximal $\theta$-pair in $\theta(M)$.

Theorem 2.5 Let $G$ be a finite group. Then $G$ is nilpotent if and only if $\overline{\theta(M) \text {, for every }} M \underset{\max }{<} G$, contains a maximal pair $(C, D)$ such that $\frac{G}{D}$ is nilpotent.

Theorem 2.6 A solvable group $G$ is nilpotent if and only if for all $M \underset{\max }{<} G$, $\theta(M)$ contains exactly one maximal pair.

There are some similar results on $\theta^{\star}$-pair that we referred to [5] and [6].

\section{Degree of $\theta$-pairs and $\theta^{\star}$-pairs}

In this section, we introduce a concept of degree for $\theta$-pairs and $\theta^{\star}$-pairs of a finite group $G$. We state the following definition.

Definition 3.1 Let $G$ be a finite group and $m(G)$ be the total number of distinct maximal subgroup of $G$. Then, $d \theta(G)$ is said to be the degree of $\theta$-pairs of $G$ and is

$$
d \theta(G)=\frac{|\theta(G)|}{m(G)} .
$$

Similarly, the degree of $\theta^{\star}$-pair of $G$ is denoted by

$$
d \theta^{\star}(G)=\frac{\left|\theta^{\star}(G)\right|}{m(G)} .
$$


We may also define the above degree for maximal $\theta$-pairs and $\theta^{\star}$-pairs of $G$. We denote them by $d \theta_{m}(G)$ and $d \theta_{m}^{\star}(G)$, respectively. In the following lemma we compare the above degrees.

Lemma 3.2 Let $G$ be a finite group $G$. then

(i) $d \theta_{m}(G) \leq d \theta(G)$;

(ii) $d \theta_{m}^{\star}(G) \leq d \theta^{\star}(G)$;

(iii) $d \theta_{m}^{\star}(G) \leq d \theta_{m}(G)$.

Proof It is clear by definitions 2.2 and 2.3.

Lemma 3.3 Let $G$ be a finite non-abelian simple group. Then $\left|\theta_{m}(G)\right|=$ 1.

Proof It is clear that $(G, 1)$ is a $\theta$-pair for every maximal subgroup $M$ of $G$. So we have to show that $(G, 1)$ is a unique maximal $\theta$-pair. If $(C, D)$ is a $\theta$-pair of a maximal subgroup of $G$, then there are two possibilities for $D$. If $D=G$, then by condition (b) of Definition 2.1, we should have $\langle M, D\rangle=M$ which is a contradiction. Thus, $D$ must be the identity subgroup. So, $(C, D)=(C, 1)$ and therefore

$\theta(M)=\{(C, 1):<C, M>=G$ and $C$ has no proper normal subgroup of $G\}$.

Since $(G, 1) \in \theta(M)$ and is the maximal element of $\theta(M)$, we have $\theta_{m}(G)=$ $\{(G, 1)\}$ and the proof is completed.

Lemma 3.4 Let $G$ be a finite non-abelian simple group. Then $m(G)>1$. Proof Assume that $m(G)=1$ and $M$ is a unique maximal subgroup of $G$. Then we should have $M^{g}=M$ for every $g \in G$ and so $M \triangleleft G$. If $M=1$, then $G$ is a cyclic group of prime order which is a contradiction. Hence $M \neq 1$ and it implies that $m(G)>1$.

Theorem 3.5 Let $G$ be a finite non-abelian simple group. Then

$$
d \theta_{m}(G)=d \theta_{m}^{\star}(G)=\frac{1}{m(G)}<1
$$

Proof By lemmas 3.2 and 3.3, the proof is clear.

The degree of $\theta$-pairs or $\theta^{\star}$-pairs may have the values equal or bigger than 1. The following example proves this fact.

\section{Example 3.6}

$\overline{\text { (i) Let } G=S_{3}}$, then we have 4 maximal subgroups

$$
M_{1}=<(12)>, M_{2}=<(13)>, M_{3}=<(23)>
$$


and $M_{4}=A_{3}$. We can easily see that

$$
\begin{aligned}
\theta(G)=\theta^{\star}(G)= & \theta_{m}(G)=\theta_{m}^{\star}(G)=\left\{\left(G, A_{3}\right),\left(A_{3}, 1\right),\right. \\
& \left.\left(M_{1}, 1\right),\left(M_{2}, 1\right),\left(M_{3}, 1\right)\right\} .
\end{aligned}
$$

Thus

$$
d \theta(G)=d \theta^{\star}(G)=d \theta_{m}(G)=d \theta_{m}^{\star}(G)=\frac{5}{4}>1
$$

(ii) Let $G=\mathbb{Z}_{3}$, then by the similar method as the previous example we can find that $d \theta_{m}(G)=d \theta_{m}^{\star}(G)=1$.

Theorem 3.7 Let $G$ be a cyclic group of prime order. Then

$$
d \theta(G)=d \theta^{\star}(G)=d \theta_{m}(G)=d \theta_{m}^{\star}(G)=1
$$

Proof It is clear that the only proper subgroup of $G$ is the identity subgroup. Thus $m(G)=1$ and if $M=1$ is a maximal subgroup of $G$, then $(G, 1)$ is the only $\theta$-pair and, of course is a $\theta^{\star}$-pair and maximal. Therefore $\theta(M)=$ $\theta_{m}(M)=\theta_{m}^{\star}(M)=\{(G, 1)\}$ and it completes the proof.

The following theorem is a comparison of the maximal $\theta$-pair degree of group $G$ and quotient group $\frac{G}{N}$.

Theorem 3.8 Let $G$ be a nilpotent group and $N$ be a normal subgroup of $G$ such that $N \subseteq \Phi(G)$. Then $d \theta_{m}(G)=d \theta_{m}\left(\frac{G}{N}\right)$.

Proof First, we prove that $m(G)=m\left(\frac{G}{N}\right)$. If $M$ is a maximal subgroup of $G$, then $\frac{M}{N}$ is a maximal subgroup of $\frac{G}{N}$, because if there exists a subgroup $\frac{H}{N}$

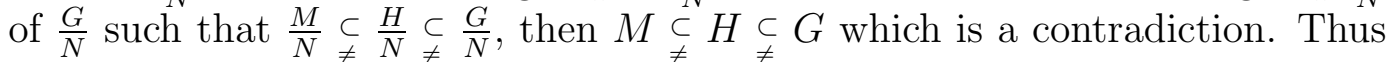
$\frac{M}{N}$ is a maximal subgroup of $\frac{G}{N}$. Conversely, if $\frac{M}{N}$ is a maximal subgroup of $\frac{G}{N}$, then by the similar method, $M$ is a maximal subgroup of $G$. Therefore, we have $m(G)=m\left(\frac{G}{N}\right)$. Now, assume that $M$ is a maximal subgroup of $G$. Then $M \triangleleft G$ and so $(G, M)$ is a $\theta$-pair of $M$ in $G$, because $\frac{G}{M}$ is simple. We can also see that $(G, M)$ is a maximal $\theta$-pair of $M$. Since, if $(C, D)$ is another maximal $\theta$-pair of $M$, then $C=G$ and so $(G, D)$ is a normal maximal $\theta$-pair. Hence, by (Theorem 2.3, [5]), $D=M_{G}$ and therefore $(C, D)=(G, M)$. It implies that $\theta_{m}(M)=\{(G, M)\}$. Now, assume that $m(G)=k$ and $M_{1}, M_{2}, \ldots, M_{k}$ are distinct maximal subgroups of $G$, then

$$
\theta_{m}(G)=\left\{\left(G, M_{1}\right),\left(G, M_{2}\right), \ldots,\left(G, M_{k}\right)\right\}
$$

By the similar way we can show that $\theta_{m}\left(\frac{M}{N}\right)=\left\{\left(\frac{G}{N}, \frac{M}{N}\right)\right\}$ and so

$$
\theta_{m}\left(\frac{G}{N}\right)=\left\{\left(\frac{G}{N}, \frac{M_{1}}{N}\right),\left(\frac{G}{N}, \frac{M_{2}}{N}\right), \ldots,\left(\frac{G}{N}, \frac{M_{k}}{N}\right)\right\} .
$$


Hence

$$
d \theta_{m}(G)=\frac{\left|\theta_{m}(G)\right|}{m(G)}=\frac{\left|\theta_{m}(G)\right|}{m\left(\frac{G}{N}\right)}=d \theta_{m}\left(\frac{G}{N}\right) .
$$

Proposition 3.9 Let $G$ be a nilpotent group. Then $d \theta_{m}(G)=1$.

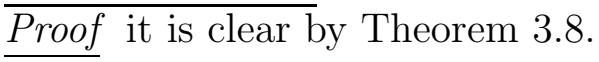

We can stablish the above result for the degree of $\theta^{\star}$-pairs and the proofs are very similar.

Finally, we state the following conjectures that we may find some evidences on it.

Conjecture 1 If $d \theta_{m}(G)=1$, then $G$ is nilpotent.

Conjecture 2 Let $G_{1}$ and $G_{2}$ be two finite groups. Then

$$
d \theta_{m}\left(G_{1} \times G_{2}\right) \leq d \theta_{m}\left(G_{1}\right) d \theta_{m}\left(G_{2}\right)
$$

\section{References}

[1] A. Ballester - Bolinches and Zho Yaoqing, on maximal subgroup of finite groups and theta pairs. Comm. in algebra, 24 (13) (1996), 41994209.

[2] J. C. Beidlemen, H. Smith, A note on supersoluble maximal subgroup and theta pair, Publ. Mat. 37 (1993) 91-94.

[3] P. Bhattacharya and N. P. Makherjee, On the intersection of a class of maximal subgroups of finite group II, J. Pure Appl. Algebra 42 (1986), 117-124.

[4] N. P. Mukherjee, P. Bhattacharya, On theta pairs for maximal subgroup, Proc. Amer. Math. Soc. 109 (1990) 585-596.

[5] Li Shirong, A note on theta pairs for maximal subgroups, Comm. in algebra, 26 (12) (1998), 4277-4284.

[6] Li Shirong and Z. Yaoqing, On theta pairs for maximal subgroups, J. Algebra 147 (2000) 133 - 142. 
[7] G. Xiuyun, On theta pairs for maximal subgroup, Comm. in algebra, 22 (12) (1994), 4653-4659.

[8] Z. Yaoqing, On theta pairs for maximal subgroup, Comm. in algebra, 23 (6) (1995), 2099-2106.

[9] Z. Yaoqing, On theta pairs for maximal subgroup on finite groups, Acta Math. 40 (1997) 97-72.

Received: June 22, 2006 